\title{
Geographic differences in allele frequencies of susceptibility SNPs for cardiovascular disease
}

Keyue Ding and Iftikhar J Kullo*

\begin{abstract}
Background: We hypothesized that the frequencies of risk alleles of SNPs mediating susceptibility to cardiovascular diseases differ among populations of varying geographic origin and that population-specific selection has operated on some of these variants.

Methods: From the database of genome-wide association studies (GWAS), we selected 36 cardiovascular phenotypes including coronary heart disease, hypertension, and stroke, as well as related quantitative traits (eg, body mass index and plasma lipid levels). We identified 292 SNPs in 270 genes associated with a disease or trait at $P<5 \times 10^{-8}$. As part of the Human Genome-Diversity Project (HGDP), 158 (54.1\%) of these SNPs have been genotyped in 938 individuals belonging to 52 populations from seven geographic areas. A measure of population differentiation, $F_{S T}$, was calculated to quantify differences in risk allele frequencies (RAFs) among populations and geographic areas.

Results: Large differences in RAFs were noted in populations of Africa, East Asia, America and Oceania, when compared with other geographic regions. The mean global $F_{\text {ST }}(0.1042)$ for 158 SNPs among the populations was not significantly higher than the mean global $F_{\text {ST }}$ of 158 autosomal SNPs randomly sampled from the HGDP database. Significantly higher global $F_{\mathrm{ST}}(P<0.05)$ was noted in eight SNPS, based on an empirical distribution of global $F_{\text {ST }}$ of 2036 putatively neutral SNPS. For four of these SNPs, additional evidence of selection was noted based on the integrated Haplotype Score.

Conclusion: Large differences in RAFs for a set of common SNPs that influence risk of cardiovascular disease were noted between the major world populations. Pairwise comparisons revealed RAF differences for at least eight SNPS that might be due to population-specific selection or demographic factors. These findings are relevant to a better understanding of geographic variation in the prevalence of cardiovascular disease.
\end{abstract}

Keywords: cardiovascular disease genetics, genome-wide association study, risk allele frequency, population differentiation

\section{Background}

Although ethnic and geographic differences in the rates of cardiovascular disease are well known $[1,2]$, the basis for such differences is not fully understood and the possible contribution of genetic factors has not been investigated. Genome-wide association studies (GWAS) have led to the identification of multiple genetic susceptibility variants (mostly single nucleotide polymorphisms, SNPs) for common diseases, such as atherosclerotic vascular disease [3]. However, most of the susceptibility SNPs were identified from populations of European ancestry.

\footnotetext{
* Correspondence: kullo.iftikhar@mayo.edu

Division of Cardiovascular Diseases, Mayo Clinic, Rochester MN 55905, USA
}

It is not known whether such SNPs also mediate susceptibility in other populations, whether the risk allele frequencies (RAFs) of these SNPs differ among the global populations, and whether these differences are due to forces of population-specific selection (ie, local selection).

An evolutionary perspective might explain why contemporary humans are at high risk for atherosclerotic vascular disease and help to better understand geographic variation in disease susceptibility [4]. Since geographically separated populations might be subject to distinctive selective environments, population-specific selection can increase population differentiation at the

\section{Biomed Central}


selected locus. However, large differences in RAFs between geographic regions are more likely to have resulted from genetic drift during population expansion after a bottleneck rather than by natural selection, a phenomenon also known as 'allelic surfing' [5-7]. From a genetic epidemiology perspective, it is of interest to explore whether RAF differences exist for susceptibility variants for cardiovascular disease, and if so, whether the differences can be accounted by allelic surfing or by natural selection.

In order to elucidate the evolutionary mechanisms that may have influenced the history and spread of genes/variants associated with cardiovascular disease and related risk factors, we calculated differences in RAFs within and out of a region of interest, and tested the population differentiation of SNPs identified in GWAS as influencing risk of cardiovascular disease. We hypothesized that SNPs mediating susceptibility to cardiovascular disease differ in frequency between human populations and may show evidence for populationspecific selection.

\section{Methods}

\section{GWAS of cardiovascular disease phenotypes and related quantitative traits}

The GWAS database [8] (accessed on Sep. $6^{\text {th }} 2009$ ) includes 211 distinct disease and traits, as well as $>1,500$ associated SNPs. From this database, we selected 36 cardiovascular diseases and traits, including coronary heart disease, stroke, and hypertension, and quantitative traits (ie, body mass index blood pressure and plasma lipid levels). A detailed description of selection of the cardiovascular disease phenotypes and related quantitative traits is provided in the Additional file 1. We identified 292 SNPs in 270 genes associated with a disease or trait at $P<5 \times 10^{-8}$. The risk allele was ascertained from original reports, eg, the allele associated with either an odds ratio $>1$ for disease phenotypes or with higher levels of body mass index, low-density lipoprotein (LDL) cholesterol, triglycerides and blood pressure, and lower levels of high-density lipoprotein (HDL) cholesterol.

\section{Genotype data}

For our population genetics analyses, we obtained genotype data from the Human Genome Diversity Project (HGDP) [9], as part of which 642,690 SNPs were genotyped in 938 unrelated individuals from 52 populations [10]. The 52 populations belong to seven geographic areas: African, Middle East, Europe, Central and South Asia, East Asia, America, and Oceania (Additional file 2). Of 292 SNPs, genotype data for 158 SNPs (54.1\%) were available in the HGDP database and analyzed in the present study (Additional file 3).

\section{Statistical analysis}

We calculated the global and regional risk allele frequency (RAF) for each SNP. For risk allele $i$ of each SNP, we computed the average $\operatorname{RAF}\left(\bar{p}_{i j}\right)$ within each geographic area $j$, as well as the difference with the average RAF computed over all other populations as $\Delta F=\bar{p}_{-i j}-\bar{p}_{i j}$, where $\bar{p}_{-i j}$ is the average RAF of allele $i$ in all populations not belonging to the geographic region $j$. We used the method of Hofer et al. [6] to randomly permute populations (10,000 times) between geographic areas and recomputed $\Delta F$ each time, to obtain its null distribution and test for the significance of $\Delta F$ for each risk allele.

The measure of population differentiation $\left(F_{\mathrm{ST}}\right)$ was calculated as described by Weir [11], using a Perl script that we had previously developed [12]. We calculated the global $F_{\mathrm{ST}}$ among 52 populations and this served as a summary of the global population differentiation. In addition, the $F_{\mathrm{ST}}$ among the seven geographical areas $(7$ $\times 7$ matrices, populations in the same geographical area were combined), and pairwise $F_{\mathrm{ST}}$ in any two populations (52 $\times 52$ matrices) were calculated. In order to test whether the mean $F_{\mathrm{ST}}$ of the 158 susceptibility SNPs was significantly different from that of random markers, we selected 158 random SNPs with allele frequency distribution similar to that of 158 susceptibility SNPs 1,000 times. The mean $F_{\mathrm{ST}}$ of the 158 susceptibility SNPs was compared to the distribution of mean $F_{\mathrm{ST}}$ of the 158 random SNPs.

To assess the statistical significance of differences in $F_{\mathrm{ST}}$ across populations, an empirical distribution of $F_{\mathrm{ST}}$ was estimated by selecting 2036 autosomal markers from HGDP database. The 2036 random markers located within intergenic regions, were selected based on the annotation tables of human chromosomes (http://genome.ucsc.edu). These regions were separated by at least $1 \mathrm{MB}$ from the closest exon, did not include centromeric regions, and provide an appropriate null distribution for spatial patterns of variation expected for putatively neutral regions [12]. Each $F_{\mathrm{ST}}$ value was compared to the corresponding empirical distribution of $F_{\mathrm{ST}}$ values to generate a $P$ value, which was corrected for minor allele frequency by comparing only to SNPs $(n=$ 2036) from the empirical distribution that fell into the same minor allele frequency bin (Pcor) [13]. For SNPs with a significantly higher global $F_{\mathrm{ST}}$, we also obtained the integrated Haplotype Score (iHS) statistic using the HGDP selection browser $[14,15]$. The iHS statistic is based on the differential levels of LD surrounding a positively selected allele compared to a background allele at the same position [16].

We assessed the geographic patterns of pairwise $F_{\mathrm{ST}}$ based on geographic distance and human migration 
routes. The method for calculating geographic distance for each pair of populations is described further in Additional file 1. A linear regression coefficient was calculated from the scatterplot of pairwise $F_{\mathrm{ST}}$ against geographic distances.

\section{Results}

\section{Distribution of risk allele frequencies (RAFs)}

We first characterized the distribution of RAFs for susceptibility SNPs for cardiovascular disease and related risk factors. The RAFs varied significantly among the populations, from being either 'fixed' $(\mathrm{RAF}=1)$ or 'missed' $(\mathrm{RAF}=0)$. A summary of the maximum differences of RAFs (ie, the largest difference of all the pairwise population comparisons) between any 2 of the 52 populations for each SNP is presented in Figure 1A. Seventy-two risk alleles were 'fixed' in at least one population whereas 86 risk alleles were 'missed' in at least one population.

We then determined the prevalence of large RAF differences between geographic regions. We tested whether populations belonging to the same geographic area have more similar RAFs than expected by chance, either due to shared demographic history or due to shared selective events, as suggested by Hofer et al. [6]. Figure 1B summarizes the distribution of differences of the average of RAFs in one of the tested geographic areas, as well as the differences with the average RAFs computed over all other populations (ie, $\Delta F=\bar{p}_{-i j}-\bar{p}_{i j}$ ). A negative $\Delta F$ indicates that the RAF is higher within the tested geographic area than in the rest of the world. We used an arbitrary threshold $(\Delta F>0.3$ or $\Delta F<-0.3)$ to define a large RAF difference [6]. Large differences in RAF between geographic areas were frequent: 69 out of 158 (43.7\%) risk alleles have $\Delta F>0.3$ or $\Delta F<-0.3$ for at least one comparison. Of the seven geographic regions, Africa, East Asia, America, and Oceania showed the largest number of loci with large RAF differences (Additional file 4). As expected under the out-of-Africa hypothesis, the differences in RAF are strikingly large when comparing Africa with the remaining geographic regions. The largest observed $\Delta F(0.731)$ was found between American and non-American populations for the SNP 'rs174570' in FADS2 (a gene that regulates unsaturation of fatty acids); the RAF in the native American population (0.048) was much lower than in the remaining populations (0.779).

\section{Global population differentiation}

We calculated the global $F_{\mathrm{ST}}$ as a measure of allele frequency differences across the 52 populations (Figure $2 \mathrm{~A}$ ). The mean global $F_{\mathrm{ST}}$ for the 158 cardiovascular susceptibility SNPs was 0.1042 , not significantly larger than the mean global $F_{\mathrm{ST}}$ of 158 randomly sampled SNPs $(P=0.064)$ (Figure $2 \mathrm{~B})$, indicating that overall, the 158 SNPs associated with cardiovascular diseases were not more differentiated than random markers. We assessed the statistical significance for each susceptibility
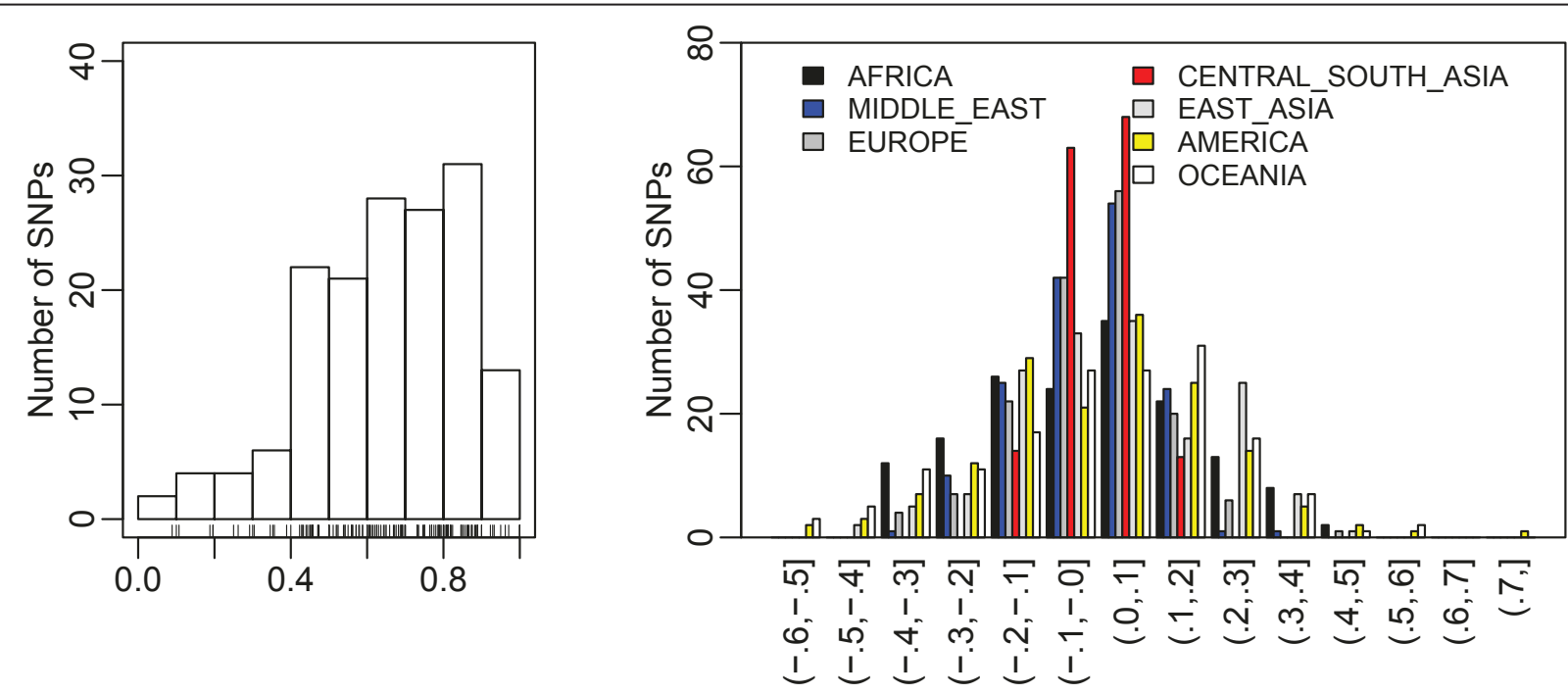

A. Max difference in RAFs between populations

B. $\Delta \mathrm{F}=\mathrm{p}_{-\mathrm{ij}}-\mathrm{p}_{\mathrm{ij}}$

Figure 1 Distribution of risk allele frequency (RAF) in HGDP populations. A. Differences in RAFs between any 2 of the 52 populations in the HGDP panel for the 158 SNPs associated with cardiovascular-diseases traits. B. Differences of RAF $\left(\Delta F=\bar{p}_{-i j}-\bar{p}_{i j}\right)$ for the comparison of a given tested region (eg, Africa) versus the rest of the world. Negative $\Delta F$ indicates that the risk allele has a higher frequency within the tested population than in the rest of the world (see methods section for the denotation of $\bar{p}_{-i j}$ and $\bar{p}_{i j}$ ). 


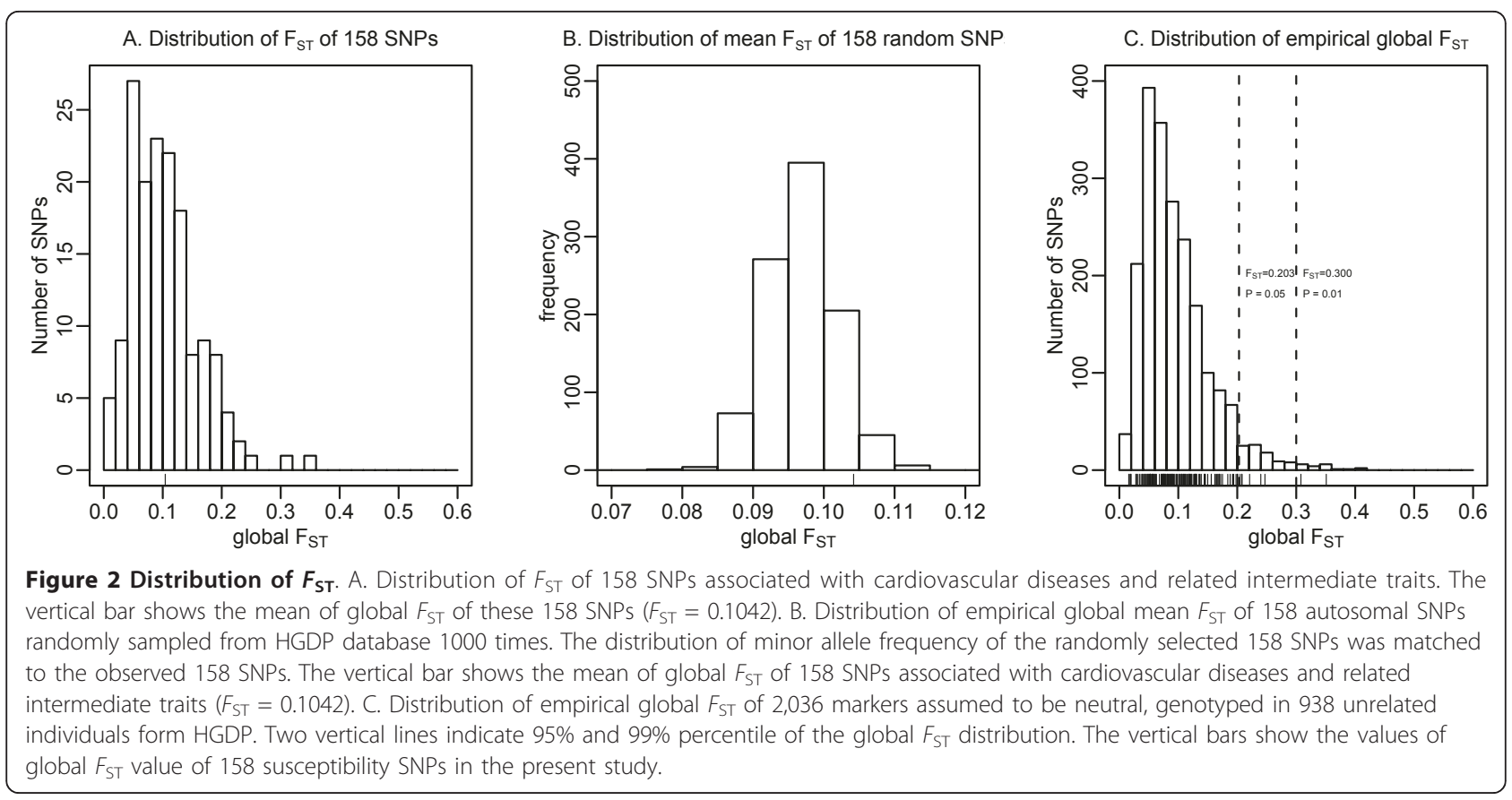

SNP by comparing its global $F_{\mathrm{ST}}$ with the empirical distribution of global $F_{\mathrm{ST}}$ for 2036 autosomal intergenic SNPs from the HGDP database (Figure 2C) [12]. Among 158 SNPs, eight SNPs had a statistically significantly higher $F_{\mathrm{ST}}\left(F_{\mathrm{ST}}>0.203 ; P<0.05\right)$ (Table 1$)$ even after correcting for allele frequency. Three of these SNPs were associated with diastolic blood pressure (rs1378942 in CSK, rs653178 in ATXN2 and rs3184504 in $S H 2 B 3$ ), two with type 1 diabetes (rs9388489 in C6orf97 and rs3184504 in SH2B3), as well as one each with a lipid trait (rs174579 in FADS2), body mass index (rs6499640 in FTO), folate pathway (rs602662 in FUT2) and inflammation (rs4796217 in CCL4L2). The world maps in Figure 3 show significant variation in the geographical distributions of the RAFs of eight SNPs with a significantly higher global $F_{\mathrm{ST}}$. Correction for minor allele frequency led to another four SNPs (rs17696736, rs2237892, rs7578597, and rs673548) reaching statistical significance (Additional file 5).

We also plotted the patterns of pairwise $F_{\mathrm{ST}}$ for these eight SNPs in any two of 52 populations and any two of seven geographic areas (Additional file 6). The patterns of population differentiation revealed the specific pattern of frequency difference of each disease-susceptibility variant among the world's populations. For example, the low RAF of rs174570 in FADS2 (associated with LDL, HDL and total cholesterol and triglycerides) in the indigenous populations of America (0.038) might contribute to the significant population differentiation between America and any of the populations in the other six geographic regions (ie, 393 (29.6\%) pairwise comparisons showed a significantly higher $\left.F_{\mathrm{ST}}\right)$. The three SNPs

Table $1 \mathrm{~A}$ list of SNPs with significantly higher global $F_{\mathrm{ST}}(P<0.05)$

\begin{tabular}{|c|c|c|c|c|c|c|}
\hline SNPs & Trait & Gene & RAF & Global $F_{\mathrm{ST}}$ & $P$ value & $P_{\text {cor }}$ \\
\hline rs1378942 & Diastolic blood pressure [17] & CSK & $0.708(\mathrm{G})$ & 0.247 & 0.025 & 0.022 \\
\hline rs653178 & Diastolic blood pressure [17] & ATXN2 & $0.177(\mathrm{G})$ & 0.205 & 0.049 & 0.031 \\
\hline rs174570 & Total, HDL and LDL cholesterol [36] & FADS2 & $0.730(C)$ & 0.351 & 0.002 & 0.000 \\
\hline rs4796217 & Macrophage-mediated inflammation [37] & $C C L 4 L 2$ & $0.425(T)$ & 0.221 & 0.040 & 0.032 \\
\hline rs602662* & Folate pathway $[22,38]$ & FUT2 & $0.294(A)$ & 0.240 & 0.027 & 0.028 \\
\hline rs9388489 & Type 1 diabetes [39] & C6orf97 & $0.634(G)$ & 0.308 & 0.008 & 0.002 \\
\hline rs3184504* & Type 1 diabetes [39]; diastolic blood pressure and systolic blood pressure[17] & $\mathrm{SH} 2 \mathrm{~B} 3$ & $0.179(\mathrm{~T})$ & 0.210 & 0.045 & 0.031 \\
\hline rs6499640 & BMI and weight[40] & FTO & $0.455(A)$ & 0.208 & 0.045 & 0.037 \\
\hline
\end{tabular}

RAF: risk allele frequency; $P_{\text {cor }} P$ value after correcting for minor allele frequency; CSK, c-src tyrosine kinase; ATXN2, ataxin 2; FADS2, fatty acid desaturase 2; CCL4L2, chemokine (C-C motif) ligand 4-like 2; FUT2, fucosyltransferase 2; C6orf97, chromosome 6 open reading frame 173; SH2B3, SH2B adaptor protein 3; FTO, fat mass and obesity associated

*, non-synonymous SNPs 


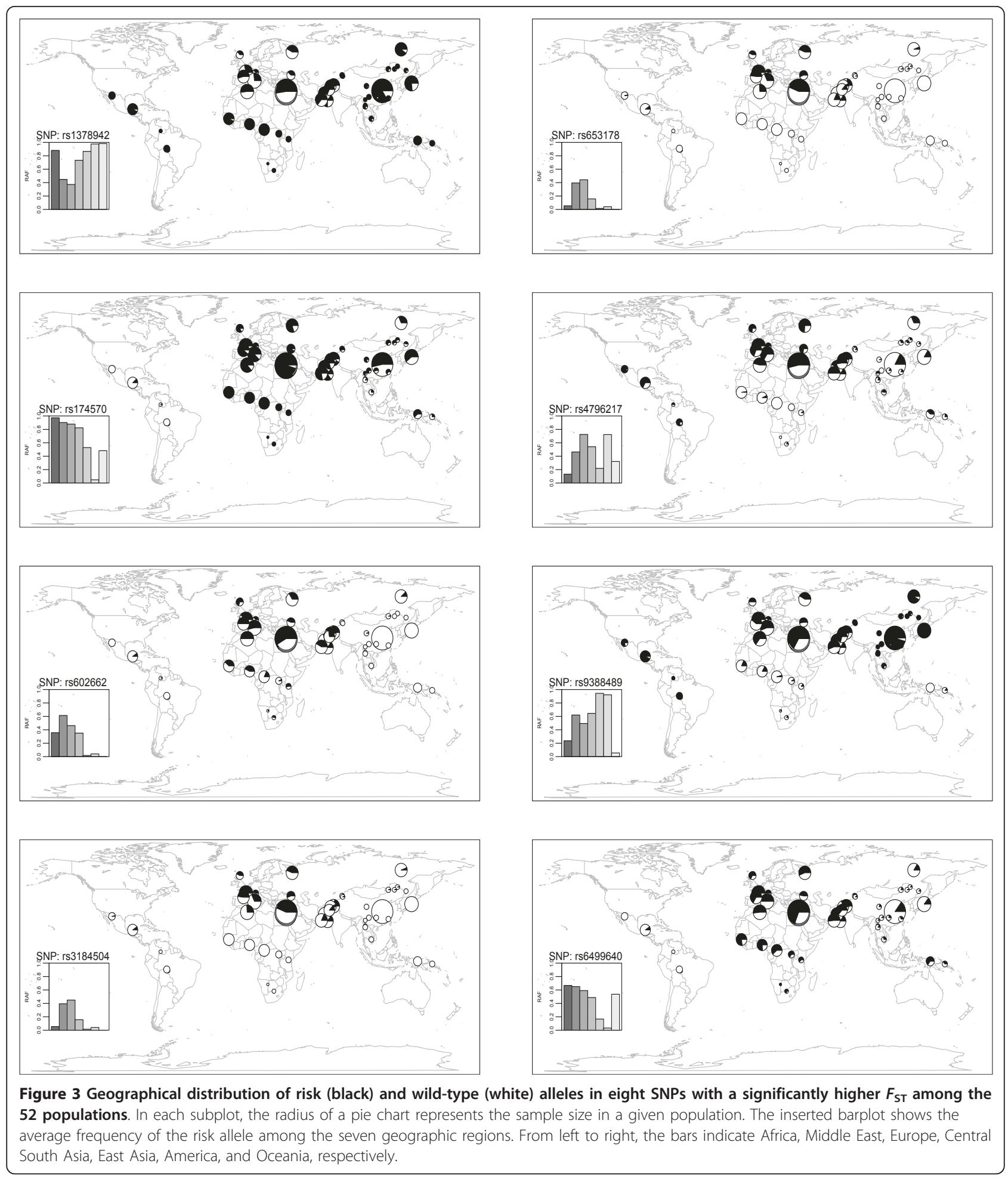

(ie, rs1378942, rs653178, and rs3184504) associated with blood pressure [17] showed different patterns of population differentiation: RAFs in rs 1378942 were $>0.5$ in most geographic regions (except in Middle East and Europe); whereas RAFs in rs653178 and rs3184504 were relatively higher in Middle East and Europe compared to the remaining geographic regions $(<0.1)$. Pairwise comparisons (in any two out of seven geographic areas) revealed differences in RAFs for more than half of the SNPs (84 out of 158) that might be due to genetic drift 
or local selection. The number of genes that showed a significantly higher $F_{\mathrm{ST}}$ in pairwise comparisons among seven geographic areas is shown in Additional file 7 . The results indicate that for risk alleles, East Asia is more differentiated from other geographic areas. Furthermore, in comparing any two of the 52 populations, for each SNP, at least one pairwise population showed a significantly higher pairwise $F_{\mathrm{ST}}$. The most undifferentiated SNP among the 52 populations was rs6511720 in $L D L R$, the RAF (0.921) being relatively high worldwide, as discussed above.

\section{Geographic patterns of population differentiation}

Genetic differentiation increases with geographic distance [18], and we therefore tested whether the $F_{\mathrm{ST}}$ (as a measure of genetic 'distance') is correlated with the geographic distance. For each SNP, a linear regression analysis between genetic distances and geographic distances between populations was performed. When combining data from 158 SNPs, the coefficient of correlation $\left(R^{2}\right)$ was $0.271\left(P<2.2 \times 10^{-16}\right)$. When each SNP was analyzed separately, all but five SNPs [ie, rs13194491, rs2383208, rs2476601, rs4900384, and rs693] showed a correlation between genetic 'distance' and geographic distance.

\section{iHS statistic}

The iHS statistic is based on the differential levels of LD surrounding a positively selected allele compared to a background allele at the same position [16]. Voight et al. [16] defined iHS $<-1.5$ or iHS $>1.5$ as suggestive evidence for natural selection, and iHS $<-2$ or iHS $>2$ as strong evidence for selection for variants that have not yet reached fixation. Using the HGDP selection browser $[14,15]$, we found that of the eight SNPs listed in Table 1, rs1378942 showed strong evidence for selection (iHS $=2.11$ in America and iHS = 2.09 in Oceania); and three SNPs showed suggestive evidence of natural selection (rs653178, iHS = 1.53 in Europe; rs9388489, iHS = 1.97 in Oceania; and rs3184504, 1.99 in Europe and 1.83 in Middle East).

\section{Discussion}

In the present study we used genotype data from 52 populations in the Human Genome Diversity Project (HGDP) to characterize the worldwide patterns of risk allele frequencies (RAFs) of 158 common SNPs associated with cardiovascular diseases and related quantitative traits. Our null hypothesis was that there is no variation of RAFs of SNPs associated with cardiovascular diseases and intermediate phenotypes, among the populations. Out of 158 susceptibility SNPs, substantial variations in RAFs of such SNPs were noted among the 52 populations, including some risk alleles being fixed or missed in at least one population. In addition we found that eight SNPs showed significant differences of RAFs among the seven geographic areas. These findings provide insights into the 'global' genetic epidemiology of cardiovascular disease.

Maximum differences in RAFs between any 2 populations ranged from 0.089 to 1.000 across SNPs with a mean of 0.661 (Figure 1A). In comparison with the rest of world, large differences in RAFs (ie, $\Delta F>0.3$ or $\Delta F<$ -0.3) were noted for the regions of Africa, East Asia, America, and Oceania (Additional file 4 and Figure 1B), consistent with the out-of-Africa hypothesis. Several explanations can be put forth for the larger differences in RAFs, including either demographic changes or shared selective events. Hofer et al. [6] suggested that large allele frequency differences between human continental groups are more likely to have occurred by genetic drift during population expansion after a bottleneck, than by selection. Using all the 158 SNPs sampled from the HGDP database, we also calculated Nei's genetic distances by 'dist.genet' function in the 'ade4' package [19] in $R$ and then used multidimensional scaling (MDS) (by 'cmdscale' function in R) to assess population differences (Additional file 8). The MDS shows differentiation among the populations corresponding to the three main clusters (ie, Europeans, Africans, and Asians). Analysis of molecular variance (AMOVA) [20] using the 'amova' function in the 'pegas' package [21] in $R$ showed that, of the total variance, $28.6 \%$ was due to variance among the seven geographic regions, $18.4 \%$ was due to variance among the populations within geographic regions, and the remaining (53.0\%) was due to variance among individuals within populations (Additional file 9).

The susceptibility variants analyzed in the present study are likely functional variants (or in linkage disequilibrium with the causal variants). The large differences in RAFs might be due to either natural selection or population demography. For example, the non-synonymous SNP rs602662 within FUT2 is in strong linkage disequilibrium with a non-sense mutation (rs601338), a plausible causal variant [22]. SNP rs3184504 is located in exon 3 of $S H 2 B 3$ which encodes the T-cell adapter protein LNK [23] and might be a causal variant (Table 1). The association of the other six SNPs has been replicated in multiple cohorts or independent samples, suggesting they are likely to be 'true' associated loci. We found additional evidence for recent positive selection (based on iHS) [16] in four (rs1378942, rs653178, rs9388489, and rs3184504) of these eight SNPs. An elevated iHS score suggests that the ancestral allele itself or the selected allele hitchhiking with the ancestral allele may be the target of selection. The iHS for each of the four SNPs was positive, indicating that the ancestral allele was under selection. 
We did not observe a significantly higher degree of population differentiation for cardiovascular disease susceptibility SNPs identified in GWAS (Figure 2B). The mean global $F_{\mathrm{ST}}$ of the 158 SNPs $(0.1042)$ was not significantly higher than the $F_{\mathrm{ST}}$ for random markers. Lohmueller et al. [24] found that 48 SNPs associated with common diseases were not significantly more differentiated across populations than random SNPs, and in another study of 25 disease-associated SNPs identified in GWAS, the mean global $F_{\mathrm{ST}}(0.100)$ was not significantly higher than random SNPs in the genome [13]. Using $F_{\mathrm{ST}}$ and iHS, Southam et al. [25] did not find consistent patterns of selection to confirm the 'thriftygenotype' hypothesis for metabolic syndrome/diabetes based on HapMap data.

It should be noted that, after correction for multiple comparison either by Bonferroni method or false discovery rate, only one SNP (rs174540) remained significant. Therefore population history and demography are likely to explain most of the difference in RAFs among populations. However, in previous studies, signatures of natural selection have also been noted in FTO (iHS = 1.991) [25], FUT2 [26], ATXN2 (high levels of LD) [27], and SH2B3 (iHS $=-2.02$ for SNP rs3184504) [15]. The non-synonymous SNP rs3184504 in SH2B3 associated with higher diastolic blood pressure (minor allele ' $\mathrm{T}$ ') may be under recent positive selection [17]. In the HapMap samples, this derived $\mathrm{T}$ allele has been shown to occur on a long haplotype ( 1.5 MB) (iHS $=-2.76, P<$ 0.006 ) [16], and local selection was noted (ie, $F_{\mathrm{ST}}=$ 0.260 for CEU-YRI comparison and $F_{\mathrm{ST}}=0.290$ for CEU-JPT/CHB comparison). The present analysis confirmed that in the HGDP sample, significant population differentiation $\left(F_{\mathrm{ST}}=0.207, P=0.048\right)$ could be attributed to a relatively higher RAF in the Middle East and Europe. A high global $F_{\mathrm{ST}}$ for rs7901695 of TCF7L2 (0.361) was noted in the HapMap samples [25], but not in the HGDP sample (0.188), possibly related to differences in sample selection between HapMap and HGDP.

Coop et al. [28] examined the role of geography and population history in the spread of selectively favored alleles, using the HapMap and HGDP databases, and argued that strong, sustained selection that drives alleles from low frequency to near fixation has been relatively rare during the past $\sim 70,000$ years [28]. The importance of geography on patterns of genetic variation has been established in previous studies [9,29-31]. We examined the prevalence of large RAFs between geographic regions and noted that RAFs of three SNPs with a significantly higher global $F_{\mathrm{ST}}$ are quite low (high)/even missed (fixed) in several populations (Figure 3). Spatial and/or temporal variation of selective pressures, such as pathogens, climate or diet, may have restricted local selection to particular populations or environments [32].
The 'ancestral-susceptibility' hypothesis for common 'complex' diseases [33] states that the ancestral allele is maladaptive in the modern environment and associated with increased disease susceptibility. We found that the ancestral allele was the risk allele in 65 out of 158 SNPs (41.1\%) based on dbSNPs and UCSC database. Thus, a subset of the cardiovascular disease susceptibility SNPs conforms to the hypothesis of 'ancestral susceptibility model' for common 'complex' diseases.

Geographic variation in the prevalence of phenotypes of medical relevance can partly be due to differential RAFs among different populations. Levels of total and LDL cholesterol in the Pima Indians (living in central and southern Arizona and Sonora (Mexico)) are lower than in populations of European origin [34]. The risk allele of rs174570 in FADS2 (a gene that regulates unsaturation of fatty acids) is missed in Pima (RAF $=0.000$ ), but fixed in Africans $(\mathrm{RAF}=1$ ) (Figure 3), raising the possibility that the low RAFs of SNPs may contribute to the low LDL cholesterol levels in Pima Indians. Pairwise comparison of RAFs among 52 populations and seven geographic areas indicated a high population differentiation between American and non-Americans, consistent with a previous study [35] showing Native Americans have greater differentiation than populations from other continental regions.

Several limitations of our study need to be mentioned. There is a potential for bias in selecting SNPs from the GWAS database as well as from the HGDP database. The SNPs were selected based on GWAS in populations of European ancestry and we were not able to characterize the patterns of geographic difference of RFs for SNPs ascertained in other specific populations, such as Africans and Asians. In addition, the majority of the HGDP populations are poorly represented in the genotyping chips and only 158 out of 292 SNPs have been genotyped in HGDP [9]. The genotyping platforms used in published GWAS varied and there was no standard threshold in declaring significant hits. Ascertainment bias in Oceania should be noted since only two populations (Papuan and NAN Melanesian) were sampled in this geographic region. Nonetheless the present study highlights a novel approach to understanding the global genetic epidemiology of cardiovascular disease, the leading cause of death worldwide and is also a step towards understanding the evolutionary genetics of this disease.

\section{Conclusions}

In conclusion, large differences in common SNPs that influence risk of cardiovascular disease were noted among the worldwide populations and are mostly due to genetic drift. The global mean $F_{\mathrm{ST}}$ for these SNPs did not differ significantly from random variants in the genome. However, pairwise comparisons revealed 
differences in RAFs in eight SNPs that might be due to local natural selection or demographic factors. These findings may help to better understand geographic variation in the prevalence of cardiovascular disease.

\section{Additional material}

Additional file 1: Supplementary methods. The supplementary methods describe 1) selection of cardiovascular disease phenotypes and related quantitative traits; and 2) calculation of geographic distance in kilometers for each pair of populations.

Additional file 2: Table S1. Sample size in the HGDP populations. Additional file 3: Table S2. A list of SNPs associated with cardiovascular diseases/traits identified in genome-wide association studies.

Additional file 4: Table S3. The number of risk alleles with various categories of RAF differences: comparisons of a given geographic area versus the rest of the world.

Additional file 5: Table S4. A list of SNPs with significantly higher global $F_{\text {ST }}($ Pcor $<0.05)$

Additional file 6: Figure S1. Pairwise comparison of population differentiation for SNPS with a significantly higher global $F_{\text {ST }}$ among 52 populations. The shaded boxes in the matrices indicate the significance level of $F_{\mathrm{ST}}$ based on the empirical distribution of the 2,036 SNPS for each pair of populations. The inserted subplot shows the comparison for the seven populations based on the geographic areas.

Additional file 7: Figure S2. Number of SNPS that showed a significantly higher $F_{S T}$ in pairwise comparisons among the seven geographic areas.

Additional file 8: Figure S3. Multidimensional scaling analysis plot (dimension I/II) of 938 individuals from seven geographic areas using 158 SNPs sampled from the HGDP database. Description: The population 'Mozabite' (black circle) was clustered into the 'Middle East', and 'Europe', which was geographically located in North Africa. The populations in 'Central_South_Asia' showed a cline from 'Middle East' and 'Europe' to 'East_Asia'. Different colors and point characters indicate seven geographic areas (see figure legend).

Additional file 9: Table S5. Analysis of molecular variance (AMOVA) of 158 SNPs.

\section{Abbreviations}

SNP: single nucleotide polymorphism; RAF: risk allele frequency; GWAS: genome-wide association study;

\section{Authors' contributions}

Conception and Design: KD and IJK; Data analyses: KD; Manuscript preparation: KD and IJK. Both authors have given final approval of the manuscript.

\section{Competing interests}

The authors declare that they have no competing interests.

Received: 23 September 2010 Accepted: 20 April 2011

Published: 20 April 2011

\section{References}

1. Keys A: Coronary heart disease in seven countries. Circulation 1970, 41(4 Suppl):1-211.

2. Anand SS, Yusuf S, Vuksan V, Devanesen S, Teo KK, Montague PA, Kelemen L, Yi C, Lonn E, Gerstein H, et al: Differences in risk factors, atherosclerosis, and cardiovascular disease between ethnic groups in Canada: the Study of Health Assessment and Risk in Ethnic groups (SHARE). Lancet 2000, 356(9226):279-284.
3. Ding K, Kullo IJ: Genome-wide association studies for atherosclerotic vascular disease and its risk factors. Circ Cardiovasc Genet 2009, 2(1):63-72.

4. Ding K, Kullo IJ: Evolutionary genetics of coronary heart disease. Circulation 2009, 119(3):459-467.

5. Excoffier L, Ray N: Surfing during population expansions promotes genetic revolutions and structuration. Trends Ecol Evol 2008, 23(7):347-351.

6. Hofer T, Ray N, Wegmann D, Excoffier L: Large allele frequency differences between human continental groups are more likely to have occurred by drift during range expansions than by selection. Ann Hum Genet 2009, 73(1):95-108.

7. Novembre J, Di Rienzo A: Spatial patterns of variation due to natural selection in humans. Nat Rev Genet 2009, 10(11):745-755.

8. Hindorff LA, Sethupathy P, Junkins HA, Ramos EM, Mehta JP, Collins FS, Manolio TA: Potential etiologic and functional implications of genomewide association loci for human diseases and traits. Proc Natl Acad Sci USA 2009, 106:9362-9367.

9. Li JZ, Absher DM, Tang H, Southwick AM, Casto AM, Ramachandran S, Cann HM, Barsh GS, Feldman M, Cavalli-Sforza LL, et al: Worldwide human relationships inferred from genome-wide patterns of variation. Science 2008, 319(5866):1100-1104.

10. The Human Genome Diversity Project Database. [ftp://ftp.cephb.fr/ hgdp_supp1].

11. Weir BS: Genetic data analysis II. Sunderland, MA: Sinauer Associated; 1986.

12. Kullo IJ, Ding K: Patterns of population differentiation of candidate genes for cardiovascular disease. BMC Genet 2007, 8:48.

13. Myles S, Davison D, Barrett J, Stoneking M, Timpson N: Worldwide population differentiation at disease-associated SNPs. BMC Med Genomics 2008, 1:22.

14. The HGDP Selection Browser. [http://hgdp.uchicago.edu/cgi-bin/gbrowse/ HGDP/].

15. Pickrell JK, Coop G, Novembre J, Kudaravalli S, Li JZ, Absher D, Srinivasan BS, Barsh GS, Myers RM, Feldman MW, et al: Signals of recent positive selection in a worldwide sample of human populations. Genome Res 2009, 19(5):826-837.

16. Voight BF, Kudaravalli $S$, Wen $X$, Pritchard JK: A map of recent positive selection in the human genome. PLoS Biol 2006, 4(3):e72.

17. Newton-Cheh C, Johnson T, Gateva V, Tobin MD, Bochud M, Coin L, Najjar SS, Zhao JH, Heath SC, Eyheramendy S, et al: Genome-wide association study identifies eight loci associated with blood pressure. Nat Genet 2009, 41:666-676.

18. Malecot G: The Mathematics of Heredity. San Francisco: Freeman; 1991

19. The ADE-4 package. [http://pbil.univ-lyon1.fr/ADE-4/].

20. Excoffier L, Smouse PE, Quattro JM: Analysis of molecular variance inferred from metric distances among DNA haplotypes: application to human mitochondrial DNA restriction data. Genetics 1992, 131(2):479-491.

21. Paradis $\mathrm{E}$ : pegas: an $\mathrm{R}$ package for population genetics with an integrated-modular approach. Bioinformatics 2010, 26(3):419-420.

22. Hazra A, Kraft P, Selhub J, Giovannucci EL, Thomas G, Hoover RN, Chanock SJ, Hunter DJ: Common variants of FUT2 are associated with plasma vitamin B12 levels. Nat Genet 2008, 40(10):1160-1162.

23. Fitau J, Boulday G, Coulon F, Quillard T, Charreau B: The adaptor molecule Lnk negatively regulates tumor necrosis factor-alpha-dependent VCAM-1 expression in endothelial cells through inhibition of the ERK1 and -2 pathways. J Biol Chem 2006, 281(29):20148-20159.

24. Lohmueller KE, Mauney MM, Reich D, Braverman JM: Variants associated with common disease are not unusually differentiated in frequency across populations. Am J Hum Genet 2006, 78(1):130-136.

25. Southam L, Soranzo N, Montgomery SB, Frayling TM, McCarthy MI, Barroso I, Zeggini E: Is the thrifty genotype hypothesis supported by evidence based on confirmed type 2 diabetes- and obesity-susceptibility variants? Diabetologia 2009, 52:1846-1851.

26. Ferrer-Admetlla A, Sikora M, Laayouni H, Esteve A, Roubinet F, Blancher A, Calafell F, Bertranpetit J, Casals F: A natural history of FUT2 polymorphism in humans. Mol Biol Evol 2009, 26:1993-2003.

27. Yu F, Sabeti PC, Hardenbol P, Fu Q, Fry B, Lu X, Ghose S, Vega R, Perez A, Pasternak $S$, et al: Positive selection of a pre-expansion CAG repeat of the human SCA2 gene. PLoS Genet 2005, 1(3):e41.

28. Coop G, Pickrell JK, Novembre J, Kudaravalli S, Li J, Absher D, Myers RM, Cavalli-Sforza LL, Feldman MW, Pritchard JK: The role of geography in human adaptation. PLoS Genet 2009, 5(6):e1000500. 
29. Ramachandran S, Deshpande O, Roseman CC, Rosenberg NA, Feldman MW Cavalli-Sforza LL: Support from the relationship of genetic and geographic distance in human populations for a serial founder effect originating in Africa. Proc Natl Acad Sci USA 2005, 102(44):15942-15947.

30. Jakobsson M, Scholz SW, Scheet P, Gibbs JR, VanLiere JM, Fung HC, Szpiech ZA, Degnan JH, Wang K, Guerreiro R, et al: Genotype, haplotype and copy-number variation in worldwide human populations. Nature 2008, 451(7181):998-1003.

31. Handley LJ, Manica A, Goudet J, Balloux F: Going the distance: human population genetics in a clinal world. Trends Genet 2007, 23(9):432-439

32. Sabeti PC, Schaffner SF, Fry B, Lohmueller J, Varilly P, Shamovsky O,

Palma A, Mikkelsen TS, Altshuler D, Lander ES: Positive natural selection in the human lineage. Science 2006, 312(5780):1614-1620.

33. Di Rienzo A, Hudson RR: An evolutionary framework for common diseases: the ancestral-susceptibility model. Trends Genet 2005, 21(11):596-601.

34. Howard BV, Davis MP, Pettitt DJ, Knowler WC, Bennett PH: Plasma and lipoprotein cholesterol and triglyceride concentrations in the Pima Indians: distributions differing from those of Caucasians. Circulation 1983, 68(4):714-724.

35. Wang S, Lewis CM, Jakobsson M, Ramachandran S, Ray N, Bedoya G, Rojas W, Parra MV, Molina JA, Gallo C, et al: Genetic variation and population structure in native Americans. PLoS Genet 2007, 3(11):e185.

36. Aulchenko YS, Ripatti S, Lindqvist I, Boomsma D, Heid IM, Pramstaller PP, Penninx BW, Janssens AC, Wilson JF, Spector T, et al: Loci influencing lipid levels and coronary heart disease risk in 16 European population cohorts. Nat Genet 2009, 41(1):47-55.

37. Melzer D, Perry JR, Hernandez D, Corsi AM, Stevens K, Rafferty I, Lauretani F, Murray A, Gibbs JR, Paolisso G, et al: A genome-wide association study identifies protein quantitative trait loci (pQTLs). PLoS Genet 2008, 4(5): e1000072.

38. Tanaka T, Scheet P, Giusti B, Bandinelli S, Piras MG, Usala G, Lai S, Mulas A, Corsi AM, Vestrini A, et al: Genome-wide association study of vitamin B6, vitamin B12, folate, and homocysteine blood concentrations. Am J Hum Genet 2009, 84(4):477-482.

39. Barrett JC, Clayton DG, Concannon P, Akolkar B, Cooper JD, Erlich HA, Julier C, Morahan G, Nerup J, Nierras C, et al: Genome-wide association study and meta-analysis find that over 40 loci affect risk of type 1 diabetes. Nat Genet 2009, 41:703-707.

40. Thorleifsson G, Walters GB, Gudbjartsson DF, Steinthorsdottir V, Sulem P, Helgadottir A, Styrkarsdottir U, Gretarsdottir S, Thorlacius S, Jonsdottir I, et al: Genome-wide association yields new sequence variants at seven loci that associate with measures of obesity. Nat Genet 2009, 41(1):18-24.

\section{Pre-publication history}

The pre-publication history for this paper can be accessed here: http://www.biomedcentral.com/1471-2350/12/55/prepub

doi:10.1186/1471-2350-12-55

Cite this article as: Ding and Kullo: Geographic differences in allele frequencies of susceptibility SNPs for cardiovascular disease. BMC Medical Genetics 2011 12:55.

\section{Submit your next manuscript to BioMed Central and take full advantage of:}

- Convenient online submission

- Thorough peer review

- No space constraints or color figure charges

- Immediate publication on acceptance

- Inclusion in PubMed, CAS, Scopus and Google Scholar

- Research which is freely available for redistribution 